\title{
Uma análise sobre as bulas de medicamentos no Brasil
}

\author{
Alexandre Martins da Cunha ${ }^{12}$, Gabriel Nascimento ${ }^{1}$, Gustavo Paiva Guedes ${ }^{1}$ \\ ${ }^{1}$ CEFET/RJ - Centro Federal de Educação Tecnológica Celso Suckow da Fonseca \\ Av. Maracanã, 229 - Rio de Janeiro - RJ - Brasil. \\ ${ }^{2}$ UFF - Universidade Federal Fluminense \\ Rua Professor Marcos Waldemar de Freitas Reis, s/n - Niterói - RJ - Brasil \\ \{alexandre.cunha, gabriel.nascimento\}@eic.cefet-rj.br, \\ gustavo.guedes@cefet-rj.br
}

\begin{abstract}
The World Health Organization states that a certain level of selfmedication may be acceptable, as long as it occurs responsibly. In this aspect, the package insert may aid in clarifying the patient's illness and treatment. However, several studies highlight problems presented in the package inserts. The present study aims to contribute by analyzing the current scenario of drug inserts in Brazil. The results show that the leaflets present on the Internet are poorly homogeneous and omit side effects. The analysis performed on a couple of package inserts on the ANVISA website indicates the existence of very technical terms and some omissions.
\end{abstract}

\begin{abstract}
Resumo. A Organização Mundial de Saúde destaca que um certo nível de automedicação pode ser aceitável, desde que ocorra de maneira responsável. Nesse aspecto, a bula do medicamento pode auxiliar no esclarecimento quanto à enfermidade e tratamento do paciente. Entretanto, diversos estudos destacam problemas apresentados nas bulas de medicamentos. O presente estudo tem o objetivo de contribuir analisando o atual cenário das bulas de medicamentos no Brasil. Os resultados indicam que as bulas presentes na internet são pouco homogêneas e omitem eventos adversos. A análise efetuada em um par de bulas do site da ANVISA indica a existência de termos muito técnicos e algumas omissões.
\end{abstract}

\section{Introdução}

O Brasil é o quinto país do mundo em número de buscas por orientação médica pela internet com o objetivo de diagnóstico e automedicação [de Oliveira et al. 2013]. A busca por informações sobre medicamentos e tratamentos médicos em sites de busca e redes sociais cresce em larga escala, o que causa preocupação para os profissionais da área de saúde [Silva and Castro 2008]. Essa preocupação é decorrente da utilização de medicamentos sem orientação médica, o que é um perigo iminente à saúde, sendo considerado um problema mundial [de Aquino et al. 2010]. Alguns problemas oriundos da automedicação são: aumento do erro no diagnóstico da doença, aparecimento de efeitos indesejáveis graves, reações alérgicas e utilização de dosagem insuficiente ou excessiva [Organization et al. 2000]. Além disso, pode mascarar os verdadeiros sintomas, comprometendo o tratamento adequado [Stimmel 1983]. 
O cenário apresentado indica que o médico deve ser a principal fonte de informação ao paciente [Silva et al. 2000]. No entanto, a Organização Mundial de Saúde (OMS) destaca que um certo nível de automedicação pode ser aceitável, desde que ocorra de forma responsável [Organization et al. 2000]. Nesse aspecto, a bula do medicamento pode auxiliar no esclarecimento quanto à enfermidade e tratamento do paciente, e portanto, é a principal fonte de informação depois do médico [Silva et al. 2000]. A presença da bula é obrigatória nos medicamentos, no entanto, apenas a inclusão da bula não é suficiente, o importante é que os pacientes sejam capazes de compreender as informações lá descritas [Da Silva et al. 2006].

$\mathrm{Na}$ tentativa de fornecer informações mais homogêneas aos pacientes, a Agência Nacional de Vigilância Sanitária (ANVISA) criou uma regulamentação pela portaria 110/97 ${ }^{1}$. Assim, as bulas de medicamentos devem conter obrigatoriamente informações de prazo de validade, cuidados de armazenamento, posologia, eventos adversos, dentre outras. No entanto, alguns estudos destacam que os indivíduos ainda possuem dificuldades na leitura das bulas, fazendo parte das principais reclamações "a linguagem muito científica" e a "excessiva quantidade de informações" [Da Silva et al. 2006, Paula et al. 2009]. Além disso, as informações nas bulas não são homogêneas, sendo notadas omissões de interações medicamentosas, erros de nomenclatura e omissão de eventos adversos [Korolkovas et al. 2006].

O cenário acima apresentado destaca diversos problemas existentes em bulas provenientes do site da ANVISA e de bulários na internet. Os bulários da internet recebem ênfase por, de maneira geral, apresentarem notória baixa qualidade [Eysenbach et al. 2002]. Embora os estudos apresentados até aqui relatem problemas já existentes entre os anos de 2000 e 2010, cabe a esse estudo uma investigação inicial sobre o atual cenário referente aos bulários na internet e às bulas existentes no site da ANVISA.

Nesse panorama, as contribuições desse estudo são: (i) construção de um conjunto de dados proveniente de um de bulário na internet, visto que não foram encontrados conjuntos de dados públicos de sites de bulários em português do Brasil; (ii) análise os eventos adversos de diferentes bulas do conjunto de dados proposto em $(i)$, de forma a verificar sua homogeneidade (e.g., omissões de eventos adversos e excesso de termos científicos); (iii) análise manualmente um par de bulas extraído do site da ANVISA, também no que se refere a homogeneidade.

O restante desse artigo está organizado da seguinte forma: a Seção 2 descreve trabalhos relacionados ao contexto de qualidade de bulários; a Seção 3 detalha o conjunto de dados produzido; a Seção 4 apresenta a metodologia utilizada para analisar os eventos adversos das bulas; a Seção 5 descreve os resultados alcançados no presente estudo e por fim, a Seção 6 conclui e apresenta alguns cenários de trabalhos futuros.

\section{Trabalhos Relacionados}

O estudo produzido em [Silva et al. 2000] se preocupa em identificar a adequação do conteúdo e da forma das bulas de medicamentos com relação a seção de Informação ao paciente. Dada a relevância da bula como referência escrita para pacientes, foram selecionadas as bulas dos medicamentos mais prescritos no ambulatório do Hospital de Clínicas

\footnotetext{
${ }^{1}$ Portaria no 110, de 10 de março de 1997 - disponível em http://www.anvisa.gov.br
} 
de Porto Alegre. Em seguida, foi realizada uma pesquisa para avaliar a existência de frases padronizadas exigidas pela portaria $110 / 97^{2}$. Também foi apresentado um formulário a alguns pacientes para a avaliação da compreensão do conteúdo das bulas, considerando paciente todo indivíduo sem formação técnico-científica. A avaliação contou com 48 bulas de 26 laboratórios, disponíveis em junho de 1998 em três farmácias de redes distintas, localizadas em Porto Alegre. Os resultados do estudo evidenciam que nenhuma das bulas atendeu, em sua totalidade, às informações exigidas por lei.

O estudo realizado em [Eysenbach et al. 2002] realiza um revisão sistemática para avaliar a dificuldade de se encontrar informações de qualidade em saúde na internet. Essa revisão utiliza como base de dados os websites MEDLINE, PREMEDLINE, Science Citation Index, Social Sciences Citation Index, Arts and Humanities Citation Index, LISA, CINAHL, PsychINFO, EMBASE, SIGLE, além de buscas pela internet, acervo pessoal e manuais. A revisão sistemática totalizou 79 estudos, 5941 sites de saúde e 1329 páginas da Web. O objetivo principal consistiu em analisar o processo de avaliação da "qualidade" de informações na área de saúde na internet. Os resultados alcançados indicam ser necessária uma padronização de critérios de qualidade, dada as diferentes abordagens adotadas nos diferentes estudos. No entanto, destacam que o risco que os indivíduos possuem de encontrar sites inadequados é uma função da proporção de informação inadequada na internet sobre a inabilidade dos indivíduos filtrarem os sites relevantes.

O estudo desenvolvido por [Segura-Bedmar and Martínez 2017] apresenta uma proposta para melhorar o vocabulário contido nas bulas de maneira automática. Para isso, foram utilizados recursos da área de PLN (Processamento de Linguagem Natural). O estudo utiliza word embeddings (em português, vetores de palavras), que são representações vetoriais capazes de capturar o valor semântico das palavras. O objetivo é encontrar o sinônimo mais simples para cada termo, utilizando como conjunto de dados o EasyDPL (Easy Drug Package Leaflets), que conta com 306 bulas e 1400 eventos adversos e sinônimos [Segura-Bedmar et al. 2016]. Os experimentos apresentam precisão de $38,5 \%$ na detecção de sinônimos.

O estudo desenvolvido neste trabalho se assemelha aos supra-citados por avaliar as informações presentes nas bulas de medicamentos. No entanto, se concentra na avaliação da homogeneidade dos eventos adversos tanto em websites quanto no site da ANVISA. Mais especificamente, se difere dos dois últimos por ser realizado em bulas na língua portuguesa.

\section{Construção do conjunto de dados}

A construção do conjunto de dados de bulas da internet foi conduzida da seguinte maneira. Inicialmente, foi efetuada uma busca no motor de busca do Google ${ }^{\mathrm{R}} 3$ utilizando a string "bulas online". Essa busca retornou milhares de resultados. No entanto, nos concentramos no primeiro resultado, ou seja, no site bulário.com.

Dessa maneira, foram extraídos 1.564 textos de bulas do site bulario.com. Os textos compreendem um total de 1.375.684 palavras e o número de termos únicos corresponde a 32.180 . O conjunto de dados se encontra nos formatos .xlsx e .json e pode ser

\footnotetext{
${ }^{2}$ Portaria $\mathrm{n}^{\mathrm{0}} 110$, de 10 de março de 1997 - disponível em http://www.anvisa.gov.br

${ }^{3}$ Buscador - Google ${ }^{\circledR}$ - www . google.com.br
} 
encontrado em https://github.com/LaCAfe/Bulario2018PT-br. Esse conjunto de dados é denominado Bulario2018PT-br.

\section{Metodologia de análise}

A metodologia de análise do presente estudo foi dividida em duas fase. A primeira fase consiste em analisar as bulas do conjunto de dados Bulario2018PT-br para evidenciar a homogeneidade das bulas presentes na internet. Dessa maneira, foi selecionada aleatoriamente uma bula desse conjunto de dados. Em seguida, foi selecionada aleatoriamente uma outra bula com o mesmo princípio ativo da primeira. Posteriormente, foi realizada uma análise manual dos eventos adversos em cada uma das bulas.

Ainda na primeira fase, foram selecionados aleatoriamente seis pares de bulas do Bulario2018PT-br para análise com o coeficiente de Jaccard. A Eq. 1 apresenta o coeficiente de Jaccard, em que $0 \leq J\left(d o c_{1}, d o c_{2}\right) \leq 1$. Essa medida corresponde à proporção do número de palavras em comum entre os documentos e o número de palavras únicas em ambos os documentos. Primeiramente, foi realizado um pre-processamento utilizando um script em Python 3, removendo a acentuação e convertendo as duas sequências de texto (strings) para letras minúsculas. Em seguida, o script calcula o coeficiente de Jaccard para essas duas strings.

$$
J\left(d o c_{1}, d o c_{2}\right)=\frac{d o c_{1} \cap d o c_{2}}{d o c_{1} \cup d o c_{2}}
$$

A segunda fase consiste em analisar os eventos adversos em um par de bulas do site da ANVISA. Para isso, foi selecionada aleatoriamente uma bula de medicamento e, em seguida, uma outra bula com o mesmo princípio ativo. Posteriormente, foi efetuada uma análise manual dos termos em cada uma das bulas.

\section{Resultados}

\subsection{Análise de bulas provenientes da internet}

A análise das bulas provenientes da internet foi efetuada com base no conjunto de dados Bulario2018P T-br. Foi selecionado aleatoriamente o medicamento Alprazolam da Medley e em seguida, o medicamento Apraz ${ }^{\circledR}$ do laboratório Hypermarcas. A Tabela 1 exibe os eventos adversos de cada um desses medicamentos. Para facilitar a compreensão, a tabela foi subdividida em três partes. Na primeira parte, são exibidos os termos em comum em ambas às bulas e, para isso, foram considerados os eventos adversos sinônimos (e.g., "perda de apetite" na bula do Alprazolam e "diminuição do apetite" na bula do Apraz). Na segunda parte, são exibidos os eventos adversos que existem na bula do Alprazolam e não existem na bula do Apraz. Por fim, na terceira parte, são apresentados os eventos adversos que existem na bula do Apraz e não existem na bula do Alprazolam. 
Tabela 1. “Eventos Adversos”dos medicamentos Alprazolam e Apraz ${ }^{\circledR}$

\begin{tabular}{ll}
\hline Alprazolam [Bulário.com 2011a] & Apraz $^{\mathbb{R}}$ [Bulário.com 2011b] \\
\hline $\begin{array}{l}\text { depressão, sonolência, perda de memória, difi- } \\
\text { culdade em controlar os movimentos do corpo, }\end{array}$ & $\begin{array}{l}\text { depresão, sonolência, alterações na memória, } \\
\text { falta de coordenação motora, fala lentificada }\end{array}$ \\
dificuldade em falar, tontura ou dor de cabeça, & e difícil de compreender, tontura, dor de \\
prisão de ventre, perda de apetite, diminuição & cabeça, prisão de ventre, diminuição do ape- \\
da libido, sensação de cabeça vazia & tite, diminuição da libido, sensação de cabeça \\
& vazia \\
\hline diarreia, aumento da líbido, problemas de & - \\
fígado, alterações do equilíbrio, aumento do & - \\
batimento cardíaco, problemas de atenção & - \\
\hline - & sedação, secura na boca, cansaço extremo, ir- \\
- & ritabilidade, confusão, confusão mental, an- \\
- & siedade, dificuldade para dormir, nervosismo, \\
- & alterações no do equilíbrio, problemas de \\
- & atenção, aumento do sono, lentidão, tremor, \\
- & visão embaçada, náusea, inflamação na pele, \\
- & impotência sexual, diminuição do peso ou au- \\
& mento do peso \\
\hline
\end{tabular}

É possível constatar que diversos eventos adversos são omitidos em ambas as bulas. Além disso, pode-se notar a não-homogeneização dos termos, visto que sinônimos são utilizados para descrever os mesmos eventos. Para apresentar uma análise mais precisa, foram analisados seis pares de bulas, selecionadas aleatoriamente, utilizando o coeficiente de Jaccard, conforme apresentado na Tabela 2. A média do coeficiente de Jaccard é indicada na última linha e corresponde a 0,27.

\begin{tabular}{ccc}
\hline Medicamento 1 & Medicamento 2 & Jaccard $(\mathrm{J})$ \\
\hline Dipirona & Novalgina & 0,13 \\
Flanax & Naproxeno & 0,31 \\
Histadin & Loratadina & 0,21 \\
Doralgina & Neosaldina & 0,32 \\
Clonazepam & Rivotril & 0,31 \\
Exodus & Espran & 0,35 \\
\hline Média & & 0,27 \\
\hline
\end{tabular}

Tabela 2. Análise dos eventos adversos em seis bulas do bulario.com.

\subsection{Análise de bulas provenientes do site da ANVISA}

A análise das bulas provenientes do site da ANVISA foi efetuada com base na seleção aleatória de um nome de medicamento a partir do Bulario2018PT-br. O medicamento selecionado foi o Citalopram (Medley). Em seguida, foi selecionado um outro medicamento com o mesmo princípio ativo (i.e., Bromidrato de Citalopram, do laboratório Nova Química). Os eventos adversos de cada um dos medicamentos é exibido na Tabela 3, que foi subdividida em quatro áreas, conforme a categoria das reações nas bulas: 
reações muito comuns (RMC), reações comuns (RC), reações incomuns (RI) e reações raras (RR). Os eventos adversos sinônimos foram exibidos em negrito. A ausência de um efeito colateral em qualquer uma das bulas foi representada como "- ".

Tabela 3. Eventos adversos dos medicamentos: Citalopram ${ }^{\circledR}$ [ANVISA 2011a] e Bromidrato de Citalopram (Nova Química). [ANVISA 2011b]

\begin{tabular}{ccc}
\hline $\begin{array}{c}\text { Tipos } \\
\text { de }\end{array}$ & Citalopram (Medley) & Bromidrato de Citalopram (Nova \\
Reações & Química)
\end{tabular}

Embora a Tabela 3 indique que alguns eventos adversos foram omitidos em ambos os medicamentos, a bula do Bromidrato de Citalopram (Nova Química) traz um aviso em negrito, informando ao paciente que se o mesmo apresentar algum dos eventos adversos abaixo, deve parar o tratamento e procurar o médico imediatamente: (a) febre alta; 
(b) agitação; (c) confusão; (d) movimentos involuntários dos músculos; (e) pele, língua, lábios ou face inchadas; (f) dificuldades em respirar ou engolir; (g) sangramentos não usuais, incluindo hemorragias gastrointestinais.

Ainda analisando a Tabela 3, pode-se notar que são utilizados sinônimos para se referir ao mesmo evento adverso (e.g., síncope e desmaio). Além disso, foram observados diversos termos técnicos (e.g., tinitus, parestesia) no Citalopram (Medley) sem a devida associação com a sua descrição. Também foi evidenciada a ausência de alguns eventos adversos (e.g., pirexia, aumento do peso). Por fim, foram encontrados diversos eventos adversos descritos de maneira diferente nas duas bulas. Por exemplo, no Bromidrato de Citalopram (Nova Química) encontramos a descrição "mulheres podem apresentar dificuldade para chegar ao orgasmo" que se difere de "orgasmo anormal em mulheres", conforme descrito na bula do Citalopram (Medley).

\section{Conclusão}

Neste estudo buscou-se investigar a situação atual da seção "eventos adversos" das bulas encontradas em websites. Para isso, foi desenvolvido um conjunto de dados contendo 1.564 bulas do site $w w w$.bulario.com. As análises realizadas com esse conjunto de dados indicam problemas na nomenclatura e na homogeneidade das bulas, mesmo com as restrições impostas pela portaria 47/20094, que rege, entre outros itens, a homogeneização das bulas. Quase uma década após a vigência da portaria, ainda é possível encontrar bulários defasados. Uma análise minuciosa foi efetuada nos medicamentos Alprazolam e o Apraz, encontrados no bulário.com. Esses medicamentos exibem diversas omissões e falta de homogeneização na descrição dos eventos adversos. Isso exige bastante atenção, dado que esses medicamentos são rotulados como tarja preta. O bulario.com informa que, entre outras fontes, utiliza o site da ANVISA como fonte de informação.

A análise efetuada em duas bulas de medicamentos provenientes do bulário da ANVISA evidenciaram a não-homogeneização das bulas, sendo por omissões de eventos adversos ou por eventos descritos ora no singular, ora no plural. Também foram encontrados diversos termos técnicos sem suas descrições, como por exemplo, hiponatremia, artralgias e mialgias. Também é importante destacar que apenas uma das bulas trouxe o aviso sobre a interrupção do tratamento caso fossem observados alguns eventos adversos.

Esse estudo abre margem para novas pesquisas que avaliem outros bulários e outros pares de bulas provenientes do site da ANVISA. Também evidencia a necessidade de uma maior homogeneização dos termos presentes nas bulas. Essa homogeneização pode ser efetuada com a criação de uma ontologia para os eventos adversos na língua portuguesa, similar à ontologia encontrada para a língua inglesa [He et al. 2014]. Esse cenário pode trazer benefícios aos pacientes e, consequentemente, à saúde pública.

\section{Referências}

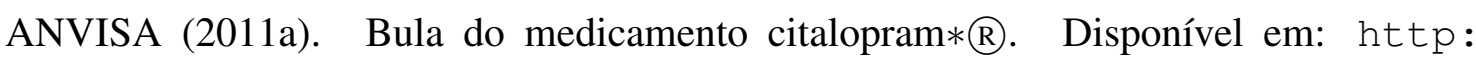
//www.anvisa.gov.br/datavisa/fila_bula/frmVisualizarBula. asp?pNuTransacao=11062252015\&pIdAnexo=3010130. Data do Acesso: 08 Mar. 2018.

\footnotetext{
${ }^{4}$ Portaria $\mathrm{n}^{\mathrm{0}} 47$, de 8 de setembro de 2009 - essa portaria se encontra disponível no endereço http://portal.anvisa.gov.br/documents/33836/2814380/RDC+47+09.pdf
} 
ANVISA (2011b). Bula do medicamentoprocimax*®. Disponível em: http: //www.anvisa.gov.br/datavisa/fila_bula/frmVisualizarBula. asp?pNuTransacao=9983572015\&pIdAnexo=2948521. Data do Acesso: 08 Mar. 2018.

Bulário.com (2011a). Bula do medicamento alprazolam. Disponível em: https: / / www. bulario.com/alprazolam. Data do Acesso: 08 Mar. 2018.

Bulário.com (2011b). Bula do medicamento apraz $囚$. Disponível em: https : / / www . bulario.com/apraz_comprimidos. Data do Acesso: 08 Mar. 2018.

Da Silva, M., Almeida, A. E. d., Oliveira, A., Correia, C., Benzatti, F., Fernandes, J., Barbosa, G., Pimenta, C., Costa, T., and Doneida, V. (2006). Estudo da bula de medicamentos: uma análise da situação. Revista de Ciências Farmacêuticas Básica e Aplicada, pages 229-236.

de Aquino, D. S., de Barros, J. A. C., and da Silva, M. D. P. (2010). A automedicação e os acadêmicos da área de saúde. Revista Ciência \& Saúde Coletiva, 15(5).

de Oliveira, F., Goloni-Bertollo, E. M., and Pavarino, É. C. (2013). A internet como fonte de informação em saúde. Journal of Health Informatics, 5(3).

Eysenbach, G., Powell, J., Kuss, O., and Sa, E.-R. (2002). Empirical studies assessing the quality of health information for consumers on the world wide web: a systematic review. Jama, 287(20):2691-2700.

He, Y., Sarntivijai, S., Lin, Y., Xiang, Z., Guo, A., Zhang, S., Jagannathan, D., Toldo, L., Tao, C., and Smith, B. (2014). Oae: the ontology of adverse events. Journal of biomedical semantics, 5(1):29.

Korolkovas, A., França, F. F. d. A. C., et al. (2006). Dicionário terapêutico guanabara. In Dicionário terapêutico guanabara.

Organization, W. H. et al. (2000). Guidelines for the regulatory assessment of medicinal products for use in self-medication.

Paula, C. d. S., Costa, C. K., Miguel, M. D., Zanin, S. M., and Spinillo, C. G. (2009). Análise crítica de bulas sob a perspectiva do usuário de medicamentos. Visão Acadêmica, 10(2).

Segura-Bedmar, I. and Martínez, P. (2017). Simplifying drug package leaflets written in spanish by using word embedding. Journal of biomedical semantics, 8(1):45.

Segura-Bedmar, I., Núnez-Gómez, L., Fernández, P. M., and Quiroz, M. (2016). Simplifying drug package leaflets. In $S M B M$, pages 20-28.

Silva, E. V. d. and Castro, L. L. C. d. (2008). A internet como forma interativa de busca de informação sobre saúde pelo paciente.

Silva, T. d., Dal-Pizzol, F., Bello, C. M., Mengue, S. S., and Schenkel, E. P. (2000). Bulas de medicamentos e a informação adequada ao paciente. Revista de Saúde Pública, 34:184-189.

Stimmel, G. (1983). Political and legal aspects of pharmacist prescribing. American Journal of Health-System Pharmacy, 40(8):1343-1344. 